\title{
Groupthink: chromosomal clustering during transcriptional memory
}

\author{
Kevin A. Morano* \\ Department of Microbiology and Molecular Genetics, University of Texas Medical School at Houston, Houston, TX 77030 USA. \\ * Corresponding Author: Kevin A. Morano; Tel: +1 713500 5890; E-mail: kevin.a.morano@uth.tmc.edu
}

Gene expression is regulated by the dynamic nature of DNA modification, histone modification, and recruitment of activating and repressing protein complexes at specific loci. Chromatin itself is further organized structurally into regions of varying accessibility, an aspect of which includes transient or sustained redistribution of portions of the genome from the nucleoplasm to the nuclear periphery. Targeting of genes to the nuclear membrane, frequently via association with components of the nuclear pore complex (NPC), has both positive and negative effects on the timing and magnitude of gene expression [1]. Moreover, chromatin localization to the nuclear membrane endows certain genes with a form of multigenerational transcriptional memory after inactivation, allowing faster reactivation in the progeny upon return to inducing conditions [2].

The mechanisms underlying these various phenomena are beginning to be understood at the molecular level, perhaps best exemplified by studies in the baker's yeast Saccharomyces cerevisiae, where precise manipulation of environmental conditions can be coupled with state of the art single-cell imaging and genetics. For example, genes have been found to localize to the nuclear periphery in response to nutrient shift (GAL1-10, INO1, SUC2, HXK1) and heat shock (HSP104, TSA2) (summarized in ref [3]). This dynamic association requires NPC proteins and, in the case of INO1, two sequence elements (gene recruitment sequence I and II; GRS) in the promoter [4]. While the role of GRSII remains unclear, GRSI recruits the Put3 transcription factor as an intermediary for NPC targeting [5]. After shift to repressing conditions, the INO1 locus remains associated with the nuclear periphery for multiple generations, conferring transcriptional memory via stabilization of a poised RNA polymerase II complex [6, 7]. This phenomenon requires a different promoter element termed the memory recruitment sequence (MRS) and a different nuclear pore protein (Nup100) than those involved in GRS recruitment [6]. Therefore, unique "DNA zip codes" dictate targeting of a gene to the nuclear periphery via distinct protein interactions. Importantly, these targeting events are independent of each other - GRSI, II and Put3 are not required during memory and the MRS is apparently dispensable for localization during gene activation [6].

The Brickner laboratory has paved the way in our understanding of these events, and elucidated much of the molecular detail. Central to their discoveries has been the development of methods to quantitatively assess subnuclear chromatin positioning. These approaches utilize DNA arrays consisting of Lac ( $\mathrm{LaCO}$ ) and Tet (TetO) Operator elements integrated at loci of interest paired with fluorescent protein-DNA binding protein fusions. Cells bearing these endogenous and/or ectopic constructs can then be visualized with confocal microscopy and intra-nuclear distances measured (i.e., gene-gene or gene-nuclear periphery) for individual cells within a population. A key observation derived from these analyses was that two copies of the same locus, for example INO1/INO1 in a diploid cell, identified with different color fluorophores, were found to cluster together in three-dimensional space [8]. Moreover, two independent loci, each bearing a GRSI element, likewise cluster during activation [8].

In the article published in this issue of Microbial Cell, Brickner and co-workers reveal that clustering also occurs during transcriptional memory [9]. Multiple aspects of INO1 memory clustering differentiate this phenomenon from that observed upon gene activation. Transcriptional memory at the GAL1-10 locus requires the nuclear basket protein Mlp1, in contrast to INO1 which targets to the NPC through Nup100 [6, 10]. Consistently, GAL1 and INO1, when differentially labeled, were found not to cluster after shift to repressing conditions, indicating specificity [9]. The MRS zip code was also shown to be required for clustering, functionally linking this feature with targeting to the nuclear periphery during memory. Fascinatingly, the MRS was seen in this study to be insufficient for memory-based clustering - both GRSI and GRSII elements are also required. The same held true for the role of the GRSI binding protein Put3 and the gene activation nuclear periphery recruitment protein Nup100 [9]. Therefore, clustering during transcriptional memory requires previous clustering during gene activation, and each process relies on its own previ-

doi: 10.15698/mic2015.12.244

Received 18.11.2015, Accepted 24.11.2015, Published 26.11.2015.

Keywords: transcriptional memory, chromatin, nuclear pore complex, DNA zip code. 
ously described components.

One of the less well understood features of gene localization at the nuclear periphery is that localization is dynamic during the cell cycle. Stress-activated genes such as INO1, GAL1 and HSP1O4 transit back into the nucleoplasm (as defined by average locus-periphery distances) during $\mathrm{S}$ phase, only to re-localize at the periphery before mitosis [11]. Strikingly, gene clustering is maintained during this period, formally distinguishing clustering from NPC/nuclear membrane localization. In contrast, genes localized at the nuclear periphery during memory remain throughout the cell cycle [7]. When Brickner et al. categorized clustering with respect to bud stage, they found that while unbudded or small-budded cells (G1/S) maintained memorybased clustering, large-budded cells (G2/M) did not, even though both INO1 loci remained associated with the nuclear periphery [9]. These events are therefore uncoupled. It is additionally interesting that despite undergoing closed mitosis (the nuclear membrane in budding yeast does not break down during mitosis and reform, as it does in most other eukaryotes [12]), chromatin association with the membrane is temporarily altered. This is in contrast to spindle pole bodies, which remain intact and inserted into the nuclear membrane, anchoring microtubules throughout the cell cycle.

This work further illuminates the complex and dynamic architecture of control of gene expression by chromatin localization, but raises even more interesting questions as it does so. Chief among these is the role of clustering with respect to targeting to the nuclear periphery. Because loci with the same DNA zip code will both cluster and target to the periphery, they appear to be localizing to the same location - possibly a specific NPC. However, clustering is retained after disengagement from the membrane, suggesting that it is not the targeted location that is specific but rather the association of different portions of chromatin. What is the fate of the nuclear pore proteins involved in nuclear membrane targeting? Do they release from the NPC with the chromatin, or release from the chromatin and remain with the NPC? The fact that clustering during gene activation is a prerequisite for clustering during transcriptional memory, while utilizing distinct DNA elements

\section{ACTIVATION}

\section{MEMORY}
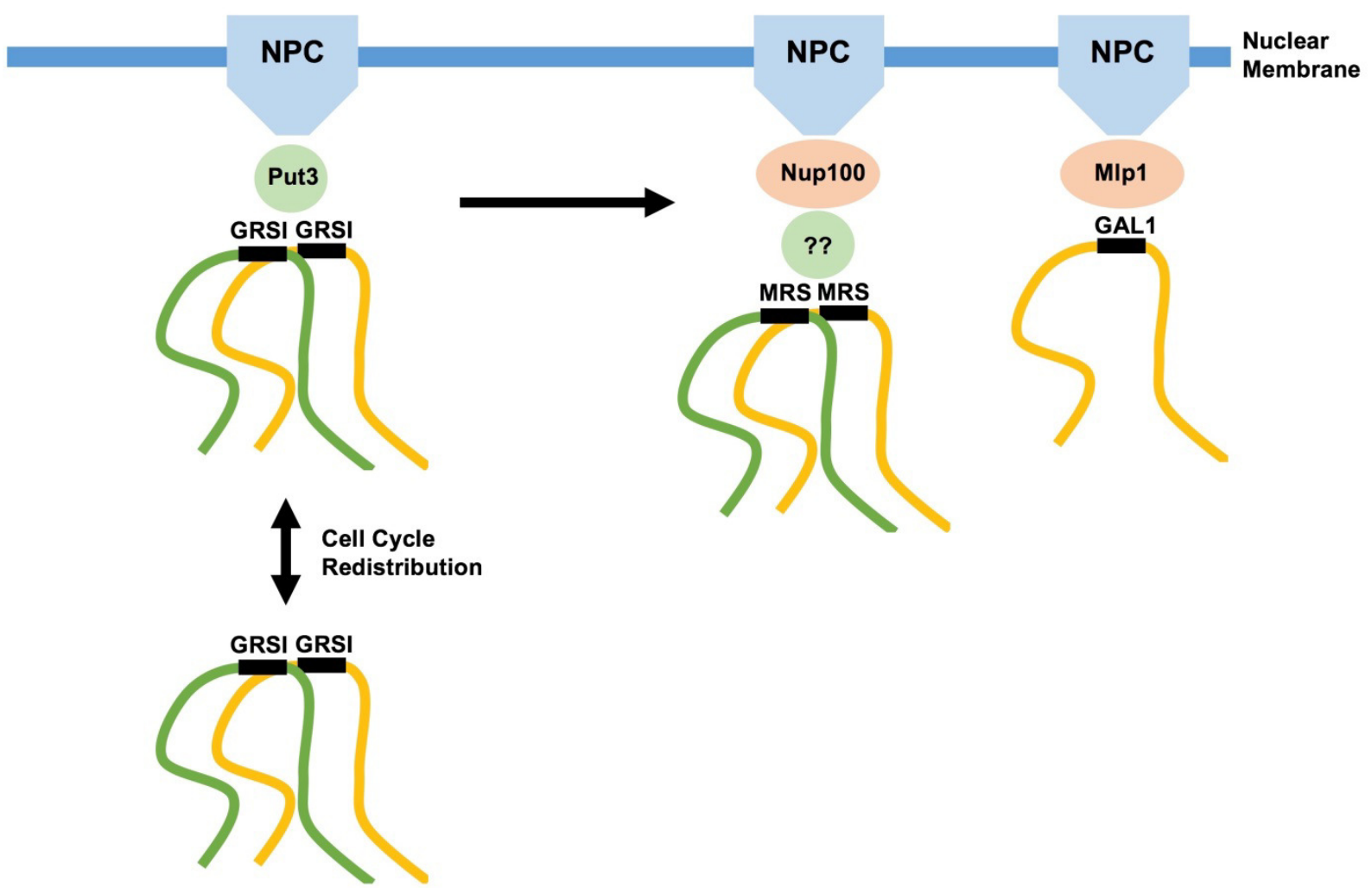

FIGURE 1: MRS-dependent interchromosomal clustering during epigenetic transcriptional memory. Portions of homologous chromosomes (yellow, green) are depicted clustering via their respective DNA zip code elements, associating with intermediary proteins and nuclear pore complexes (NPC). GRSI-mediated clustering is retained throughout the cell cycle independently of nuclear membrane binding. Memory-based clustering is specific to given genes/zip codes, possibly by virtue of distinct NPC adapter proteins. Memory-based clustering is dependent on prior clustering via GRSI elements during the activation phase. 
(and by extension, distinct DNA binding proteins) implicates a functional transfer of chromatin oversight from one system to another. It also suggests that the memory system is incapable of initiating clustering through the MRS zip code on its own. Identification of the as yet unknown MRS-binding protein (in addition to Nup100 within the NPC) may shed light on the molecular mechanisms that promote memory, defined by the rapid reinstatement of active transcription at a locus. This is thought to be achieved by stabilization of a pre-initiation transcriptional complex and a poised polymerase at the promoter, events that may be facilitated by insertion of the histone variant H2A.Z into flanking nucleosomes $[6,13]$. It is not difficult to imagine a scenario where these events are coordinated via chromatin clustering, and perhaps accelerated via association with NPC, thus increasing local concentration of key components.

As with other epigenetic regulatory mechanisms, clustering and sub-nuclear localization of specific loci allow transient or sustained modulation of gene expression in the absence of permanent nucleotide changes in the genome. Such layers of control provide an attractive target for adaptive selection - organisms capable of re-activating stress response genes faster than their neighbors are likely to gain competitive advantage, without the possible tradeoffs that arise with spontaneous mutation. Moreover, the trans-generational nature of transcriptional memory satisfies the tenet that selection can only act on heritable traits. Thus, cellular memory becomes organismal memory. Unraveling the complex language of control of gene expression via sub-nuclear positioning in yeast and other model

\section{REFERENCES}

1. Egecioglu D, Brickner JH (2011). Gene positioning and expression. Curr Opin Cell Biol 23(3): 338-345.

2. Gialitakis M, Arampatzi P, Makatounakis T, Papamatheakis J (2010). Gamma interferon-dependent transcriptional memory via relocalization of a gene locus to PML nuclear bodies. Mol Cell Biol 30(8): 2046-2056.

3. Sood V, Brickner JH (2014). Nuclear pore interactions with the genome. Curr Opin Genet Dev 25:43-49.

4. Ahmed S, Brickner DG, Light $W H$, Cajigas I, McDonough $M$, Froyshteter AB, Volpe T, Brickner JH (2010). DNA zip codes control an ancient mechanism for gene targeting to the nuclear periphery. Nat Cell Biol 12(2): 111-118.

5. Brickner DG, Ahmed S, Meldi L, Thompson A, Light W, Young M, Hickman TL, Chu F, Fabre E, Brickner JH (2012). Transcription factor binding to a DNA zip code controls interchromosomal clustering at the nuclear periphery. Dev Cell 22(6): 1234-1246.

6. Light WH, Brickner DG, Brand VR, Brickner JH (2010). Interaction of a DNA zip code with the nuclear pore complex promotes H2A.Z incorporation and INO1 transcriptional memory. Mol Cell 40(1): 112125. systems such as Drosophila may have broader impacts to human health.

Potentially, thousands of human genes exhibit association with nuclear pore proteins $[14,15]$. Human Nup98 promotes expression of developmental genes in neuronal precursor and stem cells, has been linked to transcriptional memory, and is even associated with several acute myeloid leukemias due to a chromosomal translocation fusing Nup98 with the DNA binding domain of HOXA9 [16]. Histone methylation at promoters during memory is also regulated in a manner that requires Nup98 in HeLa cells, and its homolog Nup100 in yeast $[10,15]$. As was the case with chromatin modification a decade ago, we have begun to learn the words to a new spatial positioning code. We must now learn the syntax.

\section{ACKNOWLEDGMENTS}

Work in my laboratory is supported by NIH GM074696.

\section{CONFLICT OF INTEREST}

The author declares no conflict of interest.

\section{COPYRIGHT}

(C) 2015 Morano. This is an open-access article released under the terms of the Creative Commons Attribution (CC BY) license, which allows the unrestricted use, distribution, and reproduction in any medium, provided the original author and source are acknowledged.

Please cite this article as: Kevin A. Morano (2015). Groupthink: Chromosomal clustering during transcriptional memory. Microbial Cell 2(12): 454-457. doi: 10.15698/mic2015.12.244

7. Light WH, Brickner JH (2013). Nuclear pore proteins regulate chromatin structure and transcriptional memory by a conserved mechanism. Nucleus 4(5): 357-360.

8. Brickner DG, Brickner JH (2012). Interchromosomal clustering of active genes at the nuclear pore complex. Nucleus 3(6): 487-492.

9. Brickner DG, Coukos R, Brickner JH (2015). INO1 transcriptional memory leads to DNA zip code dependent interchromosomal clustering. Microbial Cell: in press

10. Tan-Wong SM, Wijayatilake HD, Proudfoot NJ (2009). Gene loops function to maintain transcriptional memory through interaction with the nuclear pore complex. Genes Dev 23(22): 2610-2624.

11. Brickner DG, Brickner JH (2010). Cdk phosphorylation of a nucleoporin controls localization of active genes through the cell cycle. Mol Biol Cell 21(19): 3421-3432.

12. De Souza CP, Osmani SA (2007). Mitosis, not just open or closed. Eukaryot Cell 6(9): 1521-1527.

13. Brickner DG, Cajigas I, Fondufe-Mittendorf Y, Ahmed S, Lee PC, Widom J, Brickner JH (2007). H2A.Z-mediated localization of genes at the nuclear periphery confers epigenetic memory of previous transcriptional state. PLoS Biol 5(4): e81. 
14. Brown CR, Kennedy CJ, Delmar VA, Forbes DJ, Silver PA (2008). Global histone acetylation induces functional genomic reorganization at mammalian nuclear pore complexes. Genes Dev 22(5): 627-639.

15. Light WH, Freaney J, Sood V, Thompson A, D'Urso A, Horvath CM, Brickner JH (2013). A conserved role for human Nup98 in altering chromatin structure and promoting epigenetic transcriptional memory. PLoS Biol 11(3): e1001524.
16. Kasper LH, Brindle PK, Schnabel CA, Pritchard CE, Cleary ML, van Deursen JM (1999). CREB binding protein interacts with nucleoporinspecific FG repeats that activate transcription and mediate NUP98HOXA9 oncogenicity. Mol Cell Biol 19(1): 764-776. 